\title{
A Quality by Experimental Design Approach to Assess the Effect of Formulation and Process Variables on the Extrusion and Spheronization of Drug-Loaded Pellets Containing Polyplasdone ${ }^{\circledR}$ XL-10
}

\author{
Kalyan K. Saripella, ${ }^{1,2}$ Nikhil C. Loka, ${ }^{1}$ Rama Mallipeddi, ${ }^{1}$ Anuja M. Rane, ${ }^{1,3}$ and Steven H. Neau ${ }^{1,4}$
}

Received 27 February 2015; accepted 28 May 2015; published online 14 July 2015

Abstract. Successful pellet production has been reported in literature with cross-linked poly(vinylpyrrolidone), Polyplasdone ${ }^{\circledR}$ XL-10 and INF-10. In the present study, a quality by experimental design approach was used to assess several formulation and process parameter effects on the characteristics of Polyplasdone ${ }^{\circledR}$ XL-10 pellets, including pellet size, shape, yield, usable yield, friability, and number of fines. The hypothesis is that design of experiments and appropriate data analysis allow optimization of the Polyplasdone product. High drug loading was achieved using caffeine, a moderately soluble drug to allow in vitro release studies. A five-factor, two-level, half-fractional factorial design (Resolution V) with center point batches allowed mathematical modeling of the influence of the factors and their two-factor interactions on five of the responses. The five factors were Polyplasdone ${ }^{\circledR}$ level in the powder blend, volume of water in the wet massing step, wet mixing time, spheronizer speed, and spheronization time. Each factor and/or its two-factor interaction with another factor influenced pellet characteristics. The behavior of these materials under various processing conditions and component levels during extrusionspheronization have been assessed, discussed, and explained based on the results. Numerical optimization with a desirability of 0.974 was possible because curvature and lack of fit were not significant with any of the model equations. The values predicted by the optimization described well the observed responses. The hypothesis was thus supported.

KEY WORDS: crospovidone; design of experiments; extrusion; Polyplasdone; quality by design; spheronization.

\section{INTRODUCTION}

Microcrystalline cellulose (MCC) is the diluent of choice in the manufacture of pellets by extrusion-spheronization because of its water uptake capability, water holding capacity, and water yielding ability, as well as its cohesiveness and plastic behavior when wetted [1]. Since cellulose is a natural product derived from wood, it exhibits lot-to-lot variability. In addition, MCC exhibits chemical incompatibility with certain drugs [2-8]. Removal of MCC from the formulation is one approach to solve the problem. A review article addressed elimination of MCC from pellet formulations by use of appropriate alternate excipients [9], although not all alternative formulations included an active.

Crospovidone is a synthetic, cross-linked homopolymer of $\mathrm{N}$-vinyl-2-pyrrolidone that is free flowing and nonirritating

\footnotetext{
${ }^{1}$ Philadelphia College of Pharmacy, University of the Sciences, $600 \mathrm{~S}$. 43rd Street, Philadelphia, Pennsylvania 19104, USA.

${ }^{2}$ Pharma Resource Group Inc., 1005 Pontiac Road, Drexel Hill, Pennsylvania 19026, USA.

${ }^{3}$ Teva Pharmaceuticals USA Inc., 223 Quaker Road, Pomona, New York 10970, USA.

${ }^{4}$ To whom correspondence should be addressed. (e-mail: s.neau@usciences.edu)
}

[10-12]. Although the linear poly(vinylpyrrolidone) is water soluble, cross-linking leads to the hydrophilic, but water-insoluble, crospovidone. Polyplasdone ${ }^{\circledR}$ is the product name for a family of crospovidone products available from International Specialty Products (now Ashland Specialty Ingredients). The totally synthetic production of crospovidone minimizes lot-tolot variability observed with MCC [11]. A well-characterized polymer that is considered biocompatible and nontoxic, crospovidone is generally regarded as safe for use in oral dosage forms administered to humans. Because it rapidly hydrates and then causes disintegration that facilitates drug release, it serves as a superdisintegrant in solid dosage forms at $2-5 \% w / w$ levels [10-12]. The non-ionic nature of crospovidone essentially eliminates any potential ionic- or $\mathrm{pH}$-induced modifications in its behavior, such as an interaction with weak acids or bases [10, 11].

Three grades of crospovidone, namely Polyplasdone ${ }^{\circledR}$ XL, XL-10, and INF-10 in decreasing order of particle size, allowed the investigation of the particle size effects on water uptake and distribution $[13,14]$, and each of these has been a component in placebo pellets produced by extrusionspheronization [11]. Polyplasdone ${ }^{\circledR}$ XL-10 has been included as an extrusion aid at $25 \% \mathrm{w} / \mathrm{w}$ of the powder blend with lactose $\alpha$-monohydrate as the remaining material [11, 15-17], at $25 \% w / w$ with lactose $\alpha$-monohydrate and drug as the 
remaining material [18], or at $10-90 \% w / w$ polymer with drug as the remaining material in order to expand the influence of the polymer on the pellet characteristics [19]. It should be noted that lactose can dissolve in the water added during the wet mixing step and this can easily influence the product from extrusion-spheronization processing, including formation of liquid bridges in the wetted mass that contribute to the cohesive nature of the mass and the extrudate and that dry to form solid bridges in the dry pellet. The influence of the crospovidone on pellet properties might be obscured when the formulation has lactose at a substantial level.

A Box Behnken design involving Polyplasdone ${ }^{\circledR}$ XL-10 mixed with lactose $\alpha$-monohydrate (as Pharmatose ${ }^{\circledR} 200 \mathrm{M}$ ) at a fixed 1:3 ratio defined the conditions for the production of pellet batches by extrusion-spheronization [11]. The large particle version of crospovidone, Polyplasdone ${ }^{\circledR} \mathrm{XL}$, could not be used successfully in a similar production of pellets, whereas samples with smaller sized particles could produce pellets. Verheyen et al. studied the effect of drugs of differing solubility and their drug loading on the extrusionspheronization capabilities of Kollidon ${ }^{\mathbb{B}}$ CL-SF and CL-M, i.e., crospovidone grades that are manufactured by BASF (Tarrytown, NY) [19]. The wet mixing time, as well as the extrusion and spheronization conditions, were fixed; water added in the wet mixing step was adjusted to an appropriate amount for each batch. It was discovered that the yield and other pellet properties were unacceptable at drug levels higher than $60 \%$. Kollidon ${ }^{\circledR} \mathrm{CL}-\mathrm{SF}$, which is reported to have a particle size similar to that of Polyplasdone ${ }^{\circledR}$ XL-10 and INF-10 [20], failed as an extrusion-spheronization aid, whereas a micronized particle grade, Kollidon ${ }^{\circledR}$ CL-M, was successful. Jain et al. focused on the use of Polyplasdone ${ }^{\circledR}$ XL-10 as an extrusionspheronization aid in the preparation of fexofenadine hydrochloride pellets at no more than $25 \%$ of the pellet content [18]. The authors noted that higher quality pellets could be produced with a smaller median particle diameter or a higher particle size distribution of the powder blend that leads to denser packing of the particles. Reduction of spheronization plate tip speed or improved cohesiveness in the wetted mass and extrudate were also offered as suggestions to improve pellet quality, but without direction provided by the data since experimental design was not utilized. No model equation for any result as a function of the levels of the various factors has been presented, and optimization was judged only by characteristics of the batches produced. These approaches fall short because the conditions for pellet optimization could not be predicted mathematically and the optimum batch might not be produced in the experimental approach.

These studies have led to an interest in further investigation of crospovidone products, particularly for their use in extrusion-spheronization. The present study uses an experimental design approach to systematically investigate the influence of various formulation and process factors on drugloaded Polyplasdone ${ }^{\circledR}$ XL-10 pellet characteristics with Polyplasdone at high levels lest its influence be obscured by the level of a diluent used to bulk the powder mass to a consistent value. Inclusion of caffeine as a moderately soluble model drug allows assessment of drug release from the product. The hypothesis is that design of experiments and appropriate data analysis allow the influence of each factor on the responses to be assessed quantitatively. Appropriate statistical software was used to generate the experimental design, analyze the data, and provide a model equation for each response that describes quantitatively the effect of influential factors. Numerical optimization of the Polyplasdone pellets, based on the model equations, was also accomplished.

\section{MATERIALS AND METHODS}

\section{Materials}

Polyplasdone ${ }^{\circledR}$ XL-10 (27 $\mu \mathrm{m}$ average particle size, hereafter Polyplasdone) was purchased from International Specialty Products (Wayne, NJ, now Ashland Specialty Ingredients, Wilmington DE). Caffeine from Sigma Chemical Company (St. Louis, MO) served as a model drug. Distilled and de-ionized water was used as the fluid in the wet massing step.

\section{Methods}

\section{Statistical Design}

A five-factor, two-level, half-fractional factorial design with three center points (Resolution V) was generated by Design Expert $^{\circledR}$ v. 8 (StatEase, Minneapolis, MN). The five factors included the Polyplasdone content in the powder mass, the amount of water added in the wet massing step, the wet mixing time, the spheronizer speed, and the spheronization time. The levels for each of the factors in the experimental design were defined in preliminary studies and are presented in Table I. In the statistical design, the factor levels were coded for low, medium, and high settings using $-1,0$, and +1 , respectively (Table I). Center point batches represent batches where each of the factors is at its medium level. Responses included fines, total yield, usable yield, friability, aspect ratio, sphericity, and average pellet diameter, but their values are not coded. Data analysis of the influence of the coded factor levels on the actual value of the responses was accomplished using Design Expert ${ }^{\circledR}$. Influence on a particular response was considered significant at the $\alpha=0.05$ level. Once the model equations for each response were established, Design Exper ${ }^{\circledR}$ provided numerical optimization following input regarding minimizing a response, maximizing a response, or allowing the response to remain in the observed range for that response.

\section{Pellet Manufacture}

Polyplasdone and caffeine were mixed in a KitchenAid ${ }^{\circledR}$ planetary mixer for $5 \mathrm{~min}$, the amount of water as specified by the design was added, and the wet mixing time was varied between 2.5 and $5.5 \mathrm{~min}$. The wetted mass was passed through an EXDS-60 twin screw extruder (Fuji Paudal Co., Ltd., Osaka, Japan) equipped with a $1.5-\mathrm{mm}$ axial screen. The extruder speed was set at $38 \mathrm{rpm}$ to reduce the number of factors, but more importantly because, in most studies, the extruder speed does not have a statistically significant effect on pellet characteristics [21,22]. The extrudate was introduced immediately into a Q230 marumerizer ${ }^{\mathrm{TM}}$ (Fuji Paudal Co.) fitted with a cross-hatched plate with a rotational speed setting at 630,750 , or $870 \mathrm{rpm}$, providing a tip speed of $455-629 \mathrm{~m} /$ $\min$. The residence time in the spheronizer was varied between 3 and $7 \mathrm{~min}$. Pellets were collected from the spheronizer and oven dried at $60^{\circ} \mathrm{C}$ for $7 \mathrm{~h}$. 
Table I. Experimental Design Factor Levels for Polyplasdone ${ }^{\circledR}$ XL-10 Pellets

\begin{tabular}{|c|c|c|c|c|c|}
\hline \multirow[b]{2}{*}{ Levels } & \multicolumn{5}{|c|}{ Factors } \\
\hline & A & B & $\mathrm{C}$ & $\mathrm{D}$ & $\mathrm{E}$ \\
\hline & $\begin{array}{l}\text { Polyplasdone }{ }^{\circledR} \\
\text { XL-10 }(\% w / w)\end{array}$ & $\begin{array}{l}\text { Water } \\
(\mathrm{ml})\end{array}$ & $\begin{array}{l}\text { Spheronizer } \\
\text { speed (rpm) }\end{array}$ & $\begin{array}{l}\text { Spheronization } \\
\text { time }(\mathrm{min})\end{array}$ & $\begin{array}{l}\text { Wet mixing } \\
\text { time (min) }\end{array}$ \\
\hline Low $(-1)$ & 52 & 360 & 630 & 3 & 2.5 \\
\hline Medium (0) & 55 & 375 & 750 & 5 & 4.0 \\
\hline $\operatorname{High}(+1)$ & 58 & 390 & 870 & 7 & 5.5 \\
\hline
\end{tabular}

\section{Yield (Total and Usable) and Size}

The mass of dried pellets from each batch was weighed, and that weight, expressed as a percentage of the original $300 \mathrm{~g}$ dry powder mass, is reported as the total yield. Sieve analysis of the entire mass of dried pellets from each batch was conducted for $5 \mathrm{~min}$ by screening approximately $35 \mathrm{~g}$ of pellets at a time through a nest of United States Standard Sieves using a Retsch Vibrotronic VE1 sieve shaker (Brinkmann Instrument Co., Westbury, NY). The mass of the pellets retained on each sieve was measured, and the average pellet size, $d_{\mathrm{avg}}$, was calculated using the equation:

$d_{\mathrm{avg}}=\frac{\sum(\% \text { retained })(\text { average sieve aperture })}{100 \%}$

where \% retained refers to the mass of the pellets retained on a particular sieve, expressed as a percentage of the total mass of pellets analyzed. The average sieve aperture is the mean of the sieve aperture on which the mass was retained and that of the sieve above it.

For each batch, the cumulative mass of pellets in the 12/ 18 mesh cut (1.00-1.68 mm), expressed as a percentage of the total mass of pellets in that batch, was reported as the usable yield. Further characterization was conducted using only the pellets from the 12/18 mesh cut in order to reduce confounding of the other factor effects on the responses by pellet size effects. The total mass that passed through sieve No. 35 and then was collected on the pan during sieve analysis, when expressed as a percentage of the total mass of dried pellets, is reported as the fines.

\section{Scanning Electron Microscopy}

Surface characteristics and shapes of pellets were evaluated by scanning electron microscopy. Pellet samples were mounted on a metal peg with silicon adhesive and sputtercoated with gold for about 1 min using a Denton Desk II Vacuum (Moorestown, NJ). The samples could then be viewed with an S-530 Scanning Electron Microscope (Hitachi High Technologies America, Inc., Pleasanton, CA) at an accelerating voltage of $15 \mathrm{kV}$. Orion software was used to capture digital images.

\section{Friability}

Approximately $3 \mathrm{~g}$ of accurately weighed pellets were placed in a Model 1805 Roche friabilator (Vankel Industries,
Inc., Edison, $\mathrm{NJ}$ ) with 25 glass beads ( $3 \mathrm{~mm}$ in diameter). The friability test was conducted for 100 revolutions at $25 \mathrm{rpm}$. A No. 12 sieve captured the glass beads, and the pellets that passed through this sieve were collected on a No. 20 sieve. After miscellaneous smaller particles were allowed to pass through the No. 20 sieve, the remaining mass on that sieve was weighed. The friability was determined in duplicate as the percentage loss of mass of the pellets.

\section{Pellet Shape}

The shape of the pellets in a particular batch was evaluated by the QICPIC Dynamic Image Analysis System (Sympatec Inc., Clausthal-Zellerfeld, Germany) that was equipped with a RODOS/L dry dispersing unit. The highspeed dry disperser feeds the accelerated pellets at a speed of up to $100 \mathrm{~m} / \mathrm{s}$ through a Venturi tube. Images of the particles were captured by a high-speed digital camera with a synchronized, pulsed laser light source. An exposure time of approximately $1 \mathrm{~ns}$ allowed image acquisition with minimized motion blur. The images were analyzed using Windox 5.0 software. Individual pellet sphericity was calculated as the ratio of the perimeter of a circle with an area equivalent to that of the pellet image $\left(P_{\mathrm{EQPC}}\right)$ to the actual perimeter $\left(P_{\text {real }}\right)$ of the pellet image:

Sphericity $=\frac{P_{\mathrm{EQPC}}}{P_{\text {real }}}=\frac{2 \sqrt{\pi A}}{P_{\text {real }}}$

where $A$ is the area of the pellet image. The diameter of a pellet image was measured from different orientations, and the Feret diameter is defined as the largest diameter measured. The largest diameter at right angles to the Feret diameter was also assessed. The ratio of this largest diameter to the Feret diameter itself is the aspect ratio (AR) of the individual pellet image. Both sphericity and AR values are in the range $0-1$. The higher the value, the more regular is the shape of the pellet.

\section{Dissolution Studies}

Pellets containing Polyplasdone were subjected to dissolution studies using USP apparatus 2. Paddle speed was set at $100 \mathrm{rpm}$. Simulated intestinal fluid without enzymes $(0.05 \mathrm{M}$, pH 6.8 phosphate buffer) at $37^{\circ} \mathrm{C}$ was the dissolution medium. 


\section{RESULTS}

Pellet characteristics from the different batches are presented in Table II. The total yield for XL-10containing pellets ranged from 43.14 to $63.02 \% \mathrm{w} / \mathrm{w}$, indicating a substantial influence of formulation and process variables on this response. For usable yield in the 12/ 18 mesh cut, the range is 60.71 to $97.19 \% w / w$. Fines ranged from 0.10 to $6.84 \% w / w$ of the dried material recovered from the spheronizer. Sphericity values ranged from 0.91 to 0.94 , whereas the aspect ratio range was $0.77-0.94$. Average pellet diameters ranged from 1.20 to $1.55 \mathrm{~mm}$. The friability ranged from $0.28-1.56 \% \mathrm{w} / \mathrm{w}$, which is an indication of pellet ruggedness. Pellets from each batch provided immediate release of the drug.

\section{DISCUSSION}

\section{Total Yield}

Total yield was comparable or lower in this study than observed in other reports [11,19]. A lower total yield could result from the fact that another diluent was included in the formulation [15-17]. Analysis of variance (ANOVA) reports that two of the four factors in the present study, $\mathrm{C}$ (spheronizer speed) and $\mathrm{E}$ (the wet mixing time) are statistically significant $(p<0.010)$ in their influence on the total yield. Two significant interactions exist, the Polyplasdone-spheronizer speed (AC) and the spheronizer speed-spheronization time (CD) interaction $(p<0.029)$. The model equation for coded factor levels:

Total yield $=52.51-0.11 \mathrm{~A}-4.03 \mathrm{C}+1.33 \mathrm{D}$

$$
+2.05 \mathrm{E}-1.73 \mathrm{AC}-2.19 \mathrm{CD}
$$

is significant $(p=0.0005)$ and has a good fit to the data $\left(R^{2}=0.8514\right)$. Neither the lack of fit $(p=0.9074)$ nor the curvature $(p=0.8670)$ is statistically significant, indicating that the results are described well by the equation. The Normal Plot (Fig. 1a) indicates that the residuals for total yield are randomly distributed. The Residuals vs. Predicted Plot (Fig. 1b) reveals neither a trend in the residual data nor the presence of outliers. These plots for other responses are also unremarkable and, therefore, are not included in this article.

Wet mixing time (factor $\mathrm{E}$ ) appears in a positive term in the model equation and does not participate in a two-factor interaction term. For this reason, its influence on the response can be discussed as a main effect. An increase in the wet mixing time allows a longer duration over which to distribute water through the powder blend. This results in Polyplasdone that is a more consistent wetted mass that enters the extruder. The uniformity in the distribution of water should yield wet pellets that are uniform in size, such that the formation of fine particles that are lost in the gap between the plate and the spheronizer inner wall should be minimized. This positive influence of wet mixing time has also been observed previously in MCC formulations where the yield was improved with increased wet mixing times with theophylline and dyphylline but not with aminophylline [23]. This can be attributed to the fact that solubility of the drugs used in a formulation affects the distribution of water during wet massing [24]. Caffeine, a moderately soluble drug similar to dyphylline, thus requires more wet massing time to achieve uniform distribution when compared to a highly soluble drug.

The influence of the spheronizer speed on the total yield appears in the model equation as both a main factor and as a factor in a two-factor interaction (Table III). Because it is involved in a two-factor interaction term, its effect should only be interpreted in light of the other factor with which it interacts [25]. The highly abusive condition experienced at high

Table II. $2^{5-1}$ Half-Fractional Factorial Screening Design with Actual Factor Levels and Responses

\begin{tabular}{|c|c|c|c|c|c|c|c|c|c|c|c|c|}
\hline Run* & A & B & $\mathrm{C}$ & $\mathrm{D}$ & $\mathrm{E}$ & $\begin{array}{c}\text { Total } \\
\text { yield (\%) }\end{array}$ & $\begin{array}{c}\text { Usable } \\
\text { yield (\%) }\end{array}$ & $\begin{array}{c}\text { Fines } \\
(\%)\end{array}$ & $\begin{array}{c}\text { Friability } \\
(\%)\end{array}$ & $\begin{array}{l}\text { Aspect } \\
\text { ratio }\end{array}$ & Sphericity & $\begin{array}{c}D_{\text {avg }} \\
(\mathrm{mm})\end{array}$ \\
\hline 19 & -1 & -1 & -1 & -1 & 1 & 51.70 & 95.46 & 0.32 & 1.23 & 0.82 & 0.92 & 1.23 \\
\hline 2 & 1 & -1 & -1 & -1 & -1 & 53.46 & 89.72 & 0.22 & 1.56 & 0.77 & 0.91 & 1.28 \\
\hline 3 & -1 & 1 & -1 & -1 & -1 & 48.49 & 86.52 & 4.32 & 0.48 & 0.88 & 0.93 & 1.33 \\
\hline 8 & 1 & 1 & -1 & -1 & 1 & 58.24 & 89.49 & 0.71 & 0.95 & 0.92 & 0.93 & 1.27 \\
\hline 4 & -1 & -1 & 1 & -1 & -1 & 50.49 & 89.85 & 1.36 & 1.12 & 0.77 & 0.91 & 1.36 \\
\hline 5 & 1 & -1 & 1 & -1 & 1 & 50.72 & 93.84 & 0.93 & 1.34 & 0.90 & 0.94 & 1.26 \\
\hline 16 & -1 & 1 & 1 & -1 & 1 & 52.83 & 85.86 & 6.84 & 0.28 & 0.88 & 0.93 & 1.20 \\
\hline 11 & 1 & 1 & 1 & -1 & -1 & 43.14 & 89.86 & 2.21 & 1.24 & 0.89 & 0.94 & 1.31 \\
\hline 9 & -1 & -1 & -1 & 1 & -1 & 60.54 & 68.20 & 5.98 & 1.15 & 0.93 & 0.94 & 1.55 \\
\hline 14 & 1 & -1 & -1 & 1 & 1 & 63.02 & 97.20 & 0.10 & 1.23 & 0.92 & 0.94 & 1.21 \\
\hline 1 & -1 & 1 & -1 & 1 & 1 & 58.74 & 79.10 & 0.29 & 0.59 & 0.94 & 0.94 & 1.42 \\
\hline 6 & 1 & 1 & -1 & 1 & -1 & 57.72 & 84.48 & 0.38 & 0.62 & 0.86 & 0.94 & 1.35 \\
\hline 17 & -1 & -1 & 1 & 1 & 1 & 52.95 & 60.71 & 6.24 & 1.24 & 0.90 & 0.93 & 1.51 \\
\hline 13 & 1 & -1 & 1 & 1 & -1 & 44.59 & 87.82 & 0.20 & 1.48 & 0.77 & 0.91 & 1.36 \\
\hline 15 & -1 & 1 & 1 & 1 & -1 & 44.87 & 79.67 & 3.09 & 0.67 & 0.90 & 0.94 & 1.42 \\
\hline 18 & 1 & 1 & 1 & 1 & 1 & 47.90 & 76.98 & 2.64 & 0.69 & 0.88 & 0.94 & 1.43 \\
\hline 10 & 0 & 0 & 0 & 0 & 0 & 56.85 & 85.57 & 0.80 & 0.82 & 0.86 & 0.93 & 1.35 \\
\hline 7 & 0 & 0 & 0 & 0 & 0 & 48.56 & 78.56 & 1.54 & 0.95 & 0.88 & 0.94 & 1.33 \\
\hline 12 & 0 & 0 & 0 & 0 & 0 & 52.87 & 83.91 & 2.56 & 0.88 & 0.91 & 0.93 & 1.41 \\
\hline
\end{tabular}

*The runs are displayed in Standard Order. The Run designation is the order in which the batches were prepared, demonstrating the randomized order of preparation 
a



b

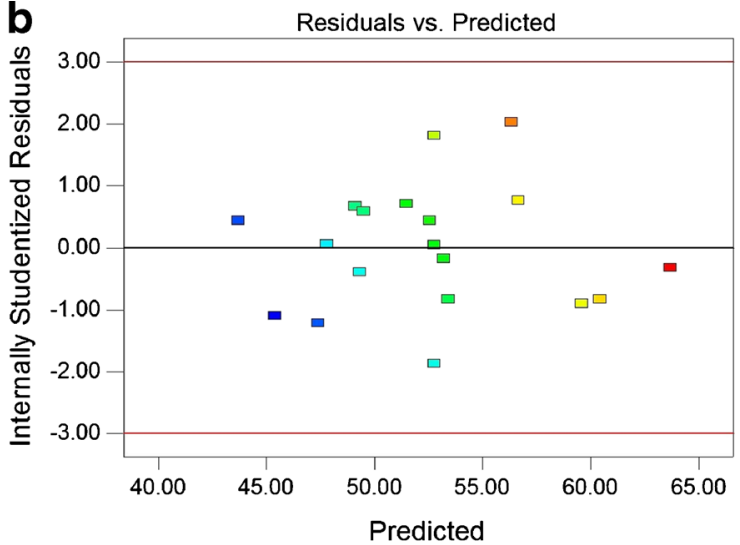

Fig. 1. Diagnostic plots for total yield: a normal plot of residuals and $\mathbf{b}$ residuals $v s$. predicted plot

spheronizer speed is likely to result in the production of fine particles that escape at the gap between the plate and the wall of the spheronizer and therefore do not contribute to total yield (Fig. 2a). The influence of the level of Polyplasdone in the formulation on the total yield is not as profoundly evident in that plot. The influence of the spheronization time on the effect of high spheronizer speed is nearly negligible, but a low spheronizer speed reveals the influence of a longer spheronization time (Fig. 2b). A longer spheronization time increases the total energy available to round up the extrudate particles, but the low spheronizer speed provides a less abusive environment, resulting in an improved total yield. A longer spheronization time at low spheronizer speed allows moist extrudate particles to aggregate with fine particles to reduce the loss of fine particles from the spheronizer.

\section{Usable Yield}

Statistical analysis revealed that four variables, namely Polyplasdone content, water level, spheronizer speed, and spheronization time, as well as multiple two-factor interactions, are statistically significant (Table III). ANOVA reports that the mathematical model:

$$
\begin{aligned}
\text { Usable yield }=84.36 & +4.00 \mathrm{~A}-0.68 \mathrm{~B}-1.60 \mathrm{C}-5.40 \mathrm{D}+0.16 \mathrm{E} \\
& -2.79 \mathrm{AB}+3.35 \mathrm{AD}-3.88 \mathrm{C} \mathrm{E}
\end{aligned}
$$

is statistically significant $(p=0.0005)$ and describes the data well $\left(R^{2}=0.8949\right)$. Although factors $\mathrm{A}$ and $\mathrm{D}$ are statistically significant in their influence on this response $(p \leq 0.0023)$, they are each involved in two-factor interactions. Discussion of

\begin{tabular}{|c|c|c|c|c|c|}
\hline Source & $\begin{array}{c}\text { Total yield } \\
p \text { values }\end{array}$ & $\begin{array}{c}\text { Usable yield } \\
p \text { values }\end{array}$ & $\begin{array}{c}\text { Fines } \\
p \text { values }\end{array}$ & $\begin{array}{c}\text { Aspect ratio } \\
p \text { values }\end{array}$ & $\begin{array}{r}\text { Friability } \\
p \text { values }\end{array}$ \\
\hline Model equation & 0.0005 & 0.0010 & $<0.0001$ & 0.0009 & $<0.0001$ \\
\hline A-Polyplasdone XL-10 & 0.8720 & 0.0031 & $<0.0001$ & 0.1729 & 0.0004 \\
\hline B-Water & $-^{a}$ & 0.5164 & 0.0864 & 0.0014 & $<0.0001$ \\
\hline C-sph speed & 0.0001 & 0.1451 & 0.0023 & 0.0772 & 0.5918 \\
\hline D-spher time & 0.0803 & 0.0004 & 0.4700 & 0.0074 & 0.2675 \\
\hline E-wet mixing time & 0.0127 & 0.8785 & 0.9100 & 0.0010 & 0.1188 \\
\hline $\mathrm{AB}$ & - & 0.0211 & - & - & - \\
\hline $\mathrm{AC}$ & 0.0287 & - & - & - & - \\
\hline $\mathrm{AD}$ & - & 0.0086 & - & 0.0014 & 0.0051 \\
\hline $\mathrm{AE}$ & - & - & - & 0.0074 & - \\
\hline $\mathrm{BC}$ & - & - & 0.0280 & - & - \\
\hline $\mathrm{BD}$ & - & - & 0.0001 & 0.0107 & - \\
\hline $\mathrm{BE}$ & - & - & - & 0.0231 & - \\
\hline $\mathrm{CD}$ & 0.0089 & - & - & 0.0107 & - \\
\hline $\mathrm{CE}$ & - & 0.0038 & $<0.0001$ & - & 0.0290 \\
\hline $\mathrm{DE}$ & - & - & - & - & - \\
\hline Curvature & 0.8670 & 0.4504 & 0.1822 & 0.3017 & 0.1573 \\
\hline Lack of Fit & 0.9074 & 0.5127 & 0.8236 & 0.8651 & 0.2406 \\
\hline$R^{2}$ & 0.8514 & 0.9010 & 0.9437 & 0.9547 & 0.9430 \\
\hline Adjusted $R^{2}$ & 0.7704 & 0.8130 & 0.8987 & 0.8901 & 0.9031 \\
\hline
\end{tabular}

Table III. ANOVA Results for Various Responses and Their Respective $p$ Values

${ }^{a}$ This indicates that this main factor or two-factor interaction is not significant and did not need to be included in the model equation for hierarchical reasons 

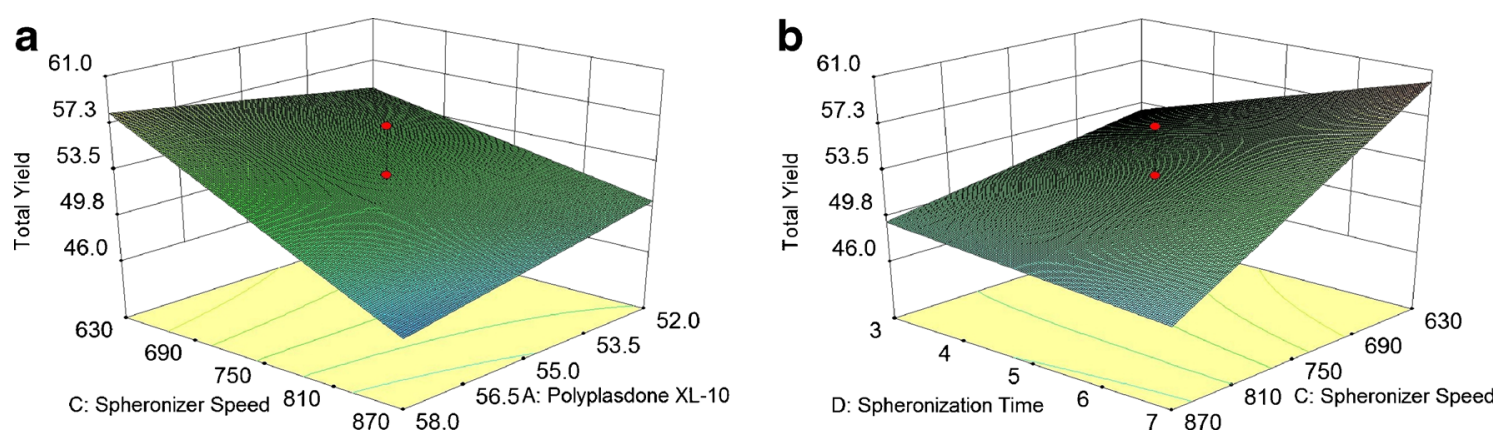

Fig. 2. Total yield as a function of $\mathbf{a}$ spheronizer speed and Polyplasdone level and $\mathbf{b}$ spheronization time and spheronizer speed

their effects on this response depends on consideration of the interaction plots for these two factors. Factors B, C, and E are retained in the model equation because each is involved in a significant two-factor interaction. Lack of fit was not statistically significant $(p=0.5122)$, suggesting that the model adequately describes this response for the factorial experiments. Curvature is not significant $(p=0.4500)$, such that it is not necessary to pursue a higher order model for this response.

In Fig. 3a, at low levels of Polyplasdone, water has little effect on the influence of Polyplasdone on usable yield. However, as the Polyplasdone level is increased, a low level of water in the wet massing step can result in a higher usable yield. A high level of both water and Polyplasdone results in a more cohesive wetted mass that allows extrudate particles to agglomerate into larger particles as they round up in the spheronizer. These larger particles exceed the usable yield size range. However, when the level of Polyplasdone is low, a reduction in the spheronization time can result in an increase in the usable yield (Fig. 3b). A longer spheronization time allows the material in the spheronizer to dry to a greater extent, and dry material can fragment into particles that are smaller than the usable yield size range.

Figure $3 \mathrm{c}$ shows that, at high spheronizer speed, the high wet mixing time results in a low usable yield. The fragmentation of extrudate into small particles at the high spheronizer speed can be exacerbated by an increase in the wet mixing time. A greater tendency for the wetted mass to dry to some extent during mixing should occur since water is available at the entire surface exposed to air. At low spheronizer speed, a less harsh environment exists in the spheronizer, such that extrudate particles should fragment to a lesser extent. Nevertheless, a low wet mixing time could result in a less uniform distribution of the water in the powder blend, resulting in regions with lower water levels in the wetted mass. Regions with lower water levels can fragment into smaller particles even at low spheronizer speed.
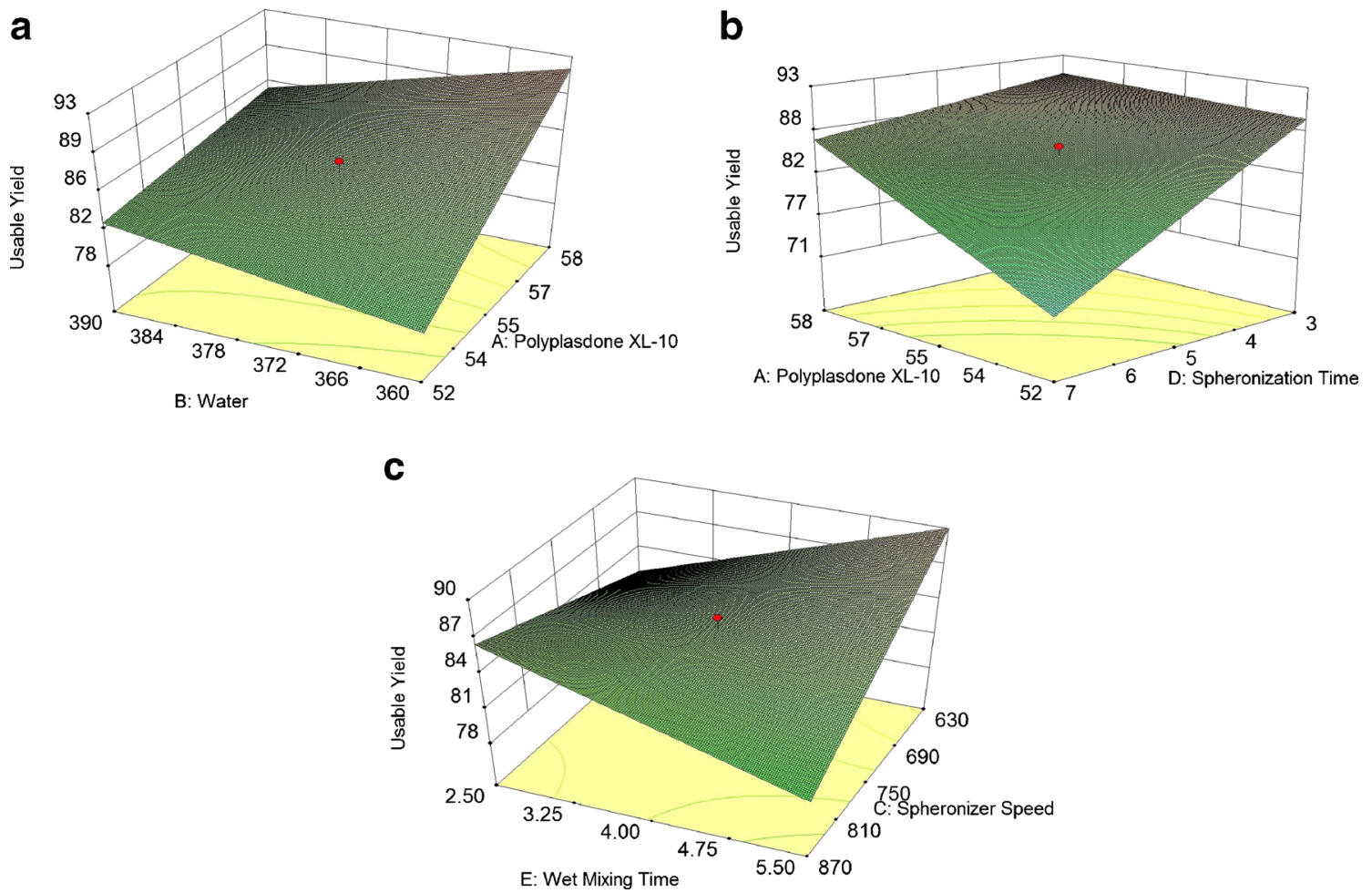

Fig. 3. Usable yield as a function of a Polyplasdone level and water, b Polyplasdone level and spheronization time, and c spheronizer speed and wet mixing time 


\section{Fines}

Across the batches, the ratio of the maximum to the minimum for fines is 68.4 and a ratio greater than 10 usually indicates that a transformation of the data is appropriate prior to ANOVA, typically because the error associated with the data does not have a normal distribution. However, after completing the data analysis without transformation of the original data, the Normal Plot revealed that the residuals were normally distributed, and the Box-Cox plot made no recommendation for a transformation (figures not shown [26]). Therefore, no data transformation was pursued. The ANOVA table (Table III) reports that the model equation is significant $(p<0.0001)$ :

Fines $=2.14-1.32 \mathrm{~A}+0.32 \mathrm{~B}+0.70 \mathrm{C}+0.13 \mathrm{D}+0.019 \mathrm{E}+0.44 \mathrm{BC}$ $-1.09 \mathrm{BD}+1.20 \mathrm{CE}$

The lack of fit $(p=0.8236)$ and the curvature $(p=0.1822)$ were not significant. All three of these suggest that the data in the design space can be described adequately by the model equation. The $R^{2}$ of 0.9437 confirms that the factorial results are well described by the model equation. With the exception of factor A, the Polyplasdone content, each main factor is involved in at least one of the two-factor interactions that is significant $(p<0.030)$, so their discussion must be approached using the two-factor interactions. Three significant two-factor interactions are reported in the equation (Table III).

Verheyen et al. reported a higher level of fines with Kollidon ${ }^{\circledR}$ CL-M than observed with microcrystalline cellulose as the extrusion aid [19]. Nevertheless, it is clear from the model equation above that an increase in the Polyplasdone level in the formulation reduces the number of fines. Adequately wetted crospovidone can act as a binder [19] to support the integrity of extrudate particles as they round up in the spheronizer and after they have dried. With a high water level, overwetted regions experience a swelling effect from the Polyplasdone and lose cohesivity. Loss of cohesivity results in the formation of fine particles in the spheronizer. This is evident in Fig. 4a where an increase in water at a high spheronizer speed can generate a profound increase in the number of fines. A high spheronization time allows fragmented pellets to become incorporated into other particles, and this occurs across the whole water range (Fig. 4b). However, the high level of water can still overhydrate some regions of the extrudate and, due to a low spheronization time, results in a greater number of fines that do not have enough time to be incorporated into other particles.

In Fig. 4c, at a high wet mixing time, the distribution of water across the powder bed should be uniform. However, as the spheronization speed is increased, a greater amount of water evaporates [27] because there is an input of more energy into the system, the air in the spheronizer moves faster, and faster moving air can carry away more water as it exits the spheronizer. The dry surfaces that result cannot allow adhesion and then incorporation of the smaller particles that would become part of the pellet under other circumstances, and the number of fines increases if the particles are too large to pass through the gap between the plate and the spheronizer wall. Even as the spheronizer speed is increased, the more uneven distribution of water in the powder bed due to a decrease in the wet mixing time reduces the number of fines. The uneven distribution of water allows a sufficient moisture level in some of the forming pellets to take up smaller particles and incorporate them, such that the overall number of fines decreases. Particles that are removed by attrition from dry surfaces, as the spheronizer speed is increased, are likely small enough to pass through the gap between the plate and the spheronizer wall and thus the fines would not increase, but the total yield would decrease.

After the pellets have been dried, small particles that have not been incorporated into a pellet, yet continue to adhere to that pellet, can break away from the surface to contribute to the level of fine particles in the batch. This can be observed with SEM images of pellets from Runs 15 and 16 (Fig. 5a, b) where the only condition that differed was the spheronization time. In Run 15, the spheronization time was high and the pellets are relatively smooth, as opposed to the images for pellets from Run 16 (Fig. 5b) where the spheronization time was low and there was insufficient time for the fragments to be incorporated into other pellets. Similar images of small particles attached to pellets were presented for hydrochlorothiazide-containing Kollidon ${ }^{\circledR}$ CL-M pellets by Verheyen et al. [19]. A longer spheronization time in the present study allows the smaller particles to become incorporated into pellets and still accomplish spheronization. This results in a higher average pellet diameter for Run 15 $(1.42 \mathrm{~mm})$ than for Run $16(1.20 \mathrm{~mm})$. These smaller particles in Fig. $5 \mathrm{~b}$ can break off the larger particle if the liquid bridges dry to weak solid bridges, and then they contribute to the fines found in the finished pellets. If there is insufficient water to cause the adherence to the larger extrudate particles, these smaller particles are not taken up on impact. Smaller particles that are not taken up can readily escape via the gap between the plate and the wall, thus causing no increase in the fines but rather a reduction in the total yield.

\section{Average Pellet Diameter}

A pellet diameter range of $1.2-1.5$ is acceptable if one wishes to avoid the problems associated with variable gastric emptying times because, as pointed out by Dressman [28], particles less than $2 \mathrm{~mm}$ in diameter exit the stomach with chyme and are not retained by the pyloric sphincter. This particle size range even allows the application of a film coat without the concern that a $2-\mathrm{mm}$ pellet diameter is exceeded.

This is a narrow range for average pellet diameter, and this is reflected in the data analysis. ANOVA provided a significant model equation $(p=0.0036)$ :

Average diameter $=1.35-0.034 \mathrm{~A}-0.0019 \mathrm{~B}+0.063 \mathrm{D}$

$$
+0.033 \mathrm{AB}-0.034 \mathrm{AD}
$$

with curvature $(p=0.6031)$ and lack of fit $(p=0.3403)$ that were not significant (Table III). It is likely that the correlation coefficient is not high $\left(R^{2}=0.7283\right)$ because the data cover a narrow range [29, 30].

Interestingly, the spheronization time had a profound effect $(p=0.0012)$ on the average pellet diameter. The premise is that the extrudate is densified during spheronization. A long 

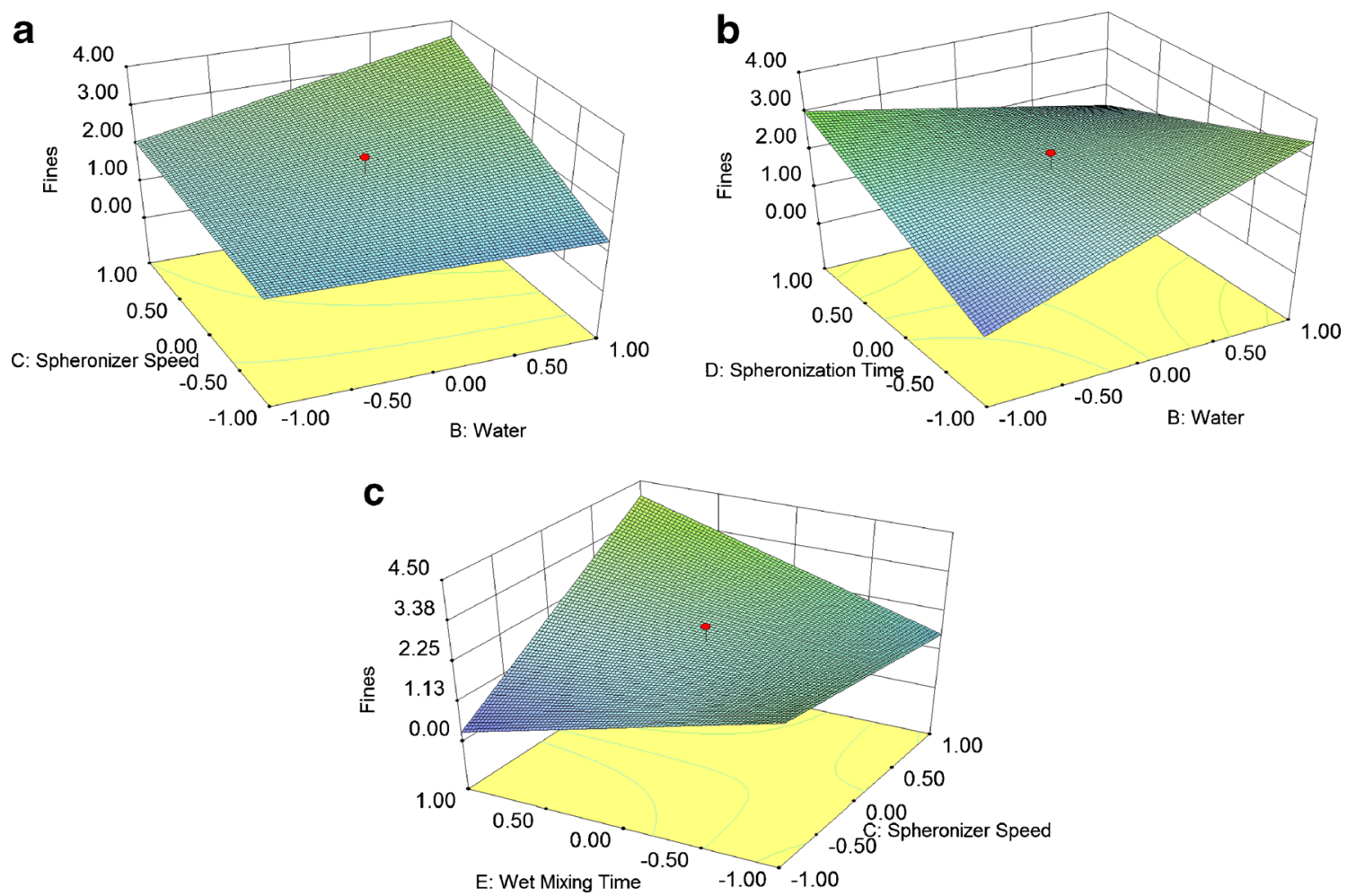

Fig. 4. Fines as a function of a water and spheronizer speed, $\mathbf{b}$ spheronization time and water, and $\mathbf{c}$ spheronizer speed and wet mixing time

spheronization time, then, densifies the extrudate to a further extent and results in a smaller pellet diameter. The opposite is predicted by the model equation (Eq. 7). It has been reported by Srujan Kumar et al. [31] that larger pellets are encouraged by longer spheronization times. Wan et al. [32] noted that an increase in pellet diameter was observed only up to a limit when increasing spheronization speed or spheronization time. Further increases in either processing parameter reduced the average pellet size.

Two interactions also exert a significant influence on the average pellet diameter, namely the Polyplasdone and water level interaction and the Polyplasdone and spheronization time interaction (figures not shown). The most profound effect is that of the Polyplasdone content when the spheronization time is high. The average diameter drops from 1.47 to $1.31 \mathrm{~mm}$ at high spheronization time as the Polyplasdone content is increased. This amounts to an $11.4 \%$ decrease in diameter that is statistically significant, but not a profound effect.

\section{Sphericity and Aspect Ratio}

Sphericity of the pellets were comparable, and the aspect ratio was somewhat improved in comparison to those values reported for Polyplsdone XL-10/lactose pellets [11]. While the


Fig. 5. Representative pellets from a Run 15 and b Run 16 
sphericity might describe the circular nature of the pellet images more clearly, the aspect ratio proved to be more discriminating regarding the influences of the different factors on the response, as observed by Mallipeddi et al. [33] with pellets where fine particle ethyl cellulose was the diluent. ANOVA of the sphericity data does not reveal the influences of the factors because the sphericity for the center point batches almost covers the breadth of the sphericity values for the entire study (Table III). This means that the random error associated with the reproduced batches with each factor at its medium level accounts for most of the variability in sphericity encountered across the whole study. Thus, quantifying the influence of a particular factor or two-factor interaction on sphericity is difficult. On the other hand, due to the greater variability across the different batches and to the similarity of the values for the center point batches, the aspect ratio can be characterized. Figure 6 presents images of pellets that correspond to two ranges of aspect ratio. At a low aspect ratio of $0.42-0.48$, the particle images proved to be oblong, whereas at a high aspect ratio of $0.78-0.85$ (comparable to the favorable properties reported by Liew et al. [11]), the particle images were close to circular. The broader range for aspect ratio facilitated characterization using ANOVA.

ANOVA revealed three main factor effects that were significant for aspect ratio (Table III), namely water level ( $p=0.0014)$, spheronization time $(p=0.0074)$, and wet mixing time $(p=0.0010)$. Factors $\mathrm{A}$ and $\mathrm{C}$ are retained in the model equation because they are involved in two-factor interactions:

Aspect ratio $=0.87-0.0068 \mathrm{~A}+0.023 \mathrm{~B}-0.0093 \mathrm{C}+0.017 \mathrm{D}+0.024 \mathrm{E}$ $-0.023 \mathrm{AD}+0.017 \mathrm{AE}-0.016 \mathrm{BD}-0.013 \mathrm{BE}-0.016 \mathrm{CD}$

Curvature was not significant $(p=0.3017)$. A lack of fit that is not significant $(p=0.8651)$ and a high $R^{2}(0.9547)$ both indicate that the model equation describes the data well.

In Fig. 7a, the highest aspect ratio occurs at high spheronization time and low Polyplasdone level. Since it is a comparable amount of water with less crospovidone, the crospovidone in the formulation is wetted to a greater extent and the crospovidone is plasticized well. An increase in the spheronization time allows more time for rounding up of these plasticized polymer particles. In Fig. 7b, an increased Polyplasdone content and wet mixing time dramatically lowers the aspect ratio. The increase in the Polyplasdone level in the formulation results in the amount of water spread across more crospovidone molecules. The reduction in the wet mixing time suggests that the water added to produce the wetted mass is not as well distributed across the powder blend. This caused variations in the level of wetting of crospovidone particles. With less water available for crospovidone, the extrudate is less plasticized [34] and less cohesive. Rowe noted that rounding up in the spheronizer is not facilitated if the polymer is not plasticized [35].

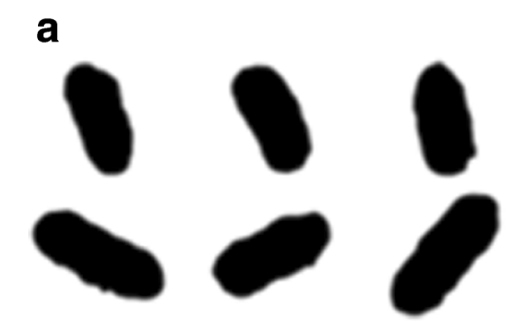

Figure $7 \mathrm{c}$ reveals the detrimental effects of an extended exposure to a highly abusive environment with the lowest aspect ratio evident when the spheronizer speed and spheronization time are both high. This abusive environment results in fragmentation that provides the smaller particles that adhere to larger particles to produce the pellets similar to those from Run 16 presented in Fig. 5b. With fixed spheronizer speed and spheronization time, Verheyen et al. observed similar small particles adhered to hydrochlorothiazide pellets at a higher drug content of 50 and $60 \% w / w$ [19]. A decrease in the spheronizer speed at high spheronization time in the present study improves the aspect ratio by providing a less abusive environment that is less likely to provide the energy necessary to further fragment the extrudate particles and less likely to dry the material further in the spheronizer. At low spheronizer speed, extending the spheronization time can provide ample opportunity to round up the material with a spheronizer speed adequate to the task. It has been suggested by Sinha et al. [36] that an attrition force resulted in fragments lost from the surface of extrudate particles, but eventually, the material is sufficiently compacted that further attrition does not occur. They also noted that rounding up takes place over a finite time and further time in the spheronizer is unnecessary.

\section{Friability}

The twenty-five glass beads included in the friability test provide a highly abusive environment for the pellets. ANOVA revealed that the Polyplasdone level in the formulation and the level of water used in the wet mixing step both provide significant main effects on friability $(p<0.0004)$ (Table III). In addition, the two-factor interactions between Polyplasdone level and spheronization time (AD, $p=0.0051)$ and between spheronizer speed and the wet mixing time $(\mathrm{CE}, p=0.0290)$ also have significant influences on this response.

$$
\begin{aligned}
\text { Friability }= & 0.99+0.15 \mathrm{~A}-0.30 \mathrm{~B}+0.016 \mathrm{C}-0.033 \mathrm{D} \\
& -0.048 \mathrm{E}-0.10 \mathrm{AD}-0.072 \mathrm{CE}
\end{aligned}
$$

The significant model equation $(p<0.0001)$ and curvature that is not significant $(p=0.1573)$ indicate that a higher order model equation is not necessary to describe well this response in this design space. A high correlation coefficient $\left(R^{2}=0.9430\right)$ and a lack of fit that is not significant $(p=0.2406)$ confirm this.

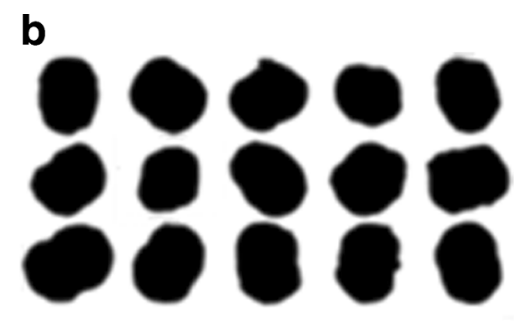

Fig. 6. Images of pellets representative of those found in a particular aspect ratio range: a $0.42-0.48$ and $\mathbf{b} 0.78-0.85$ 

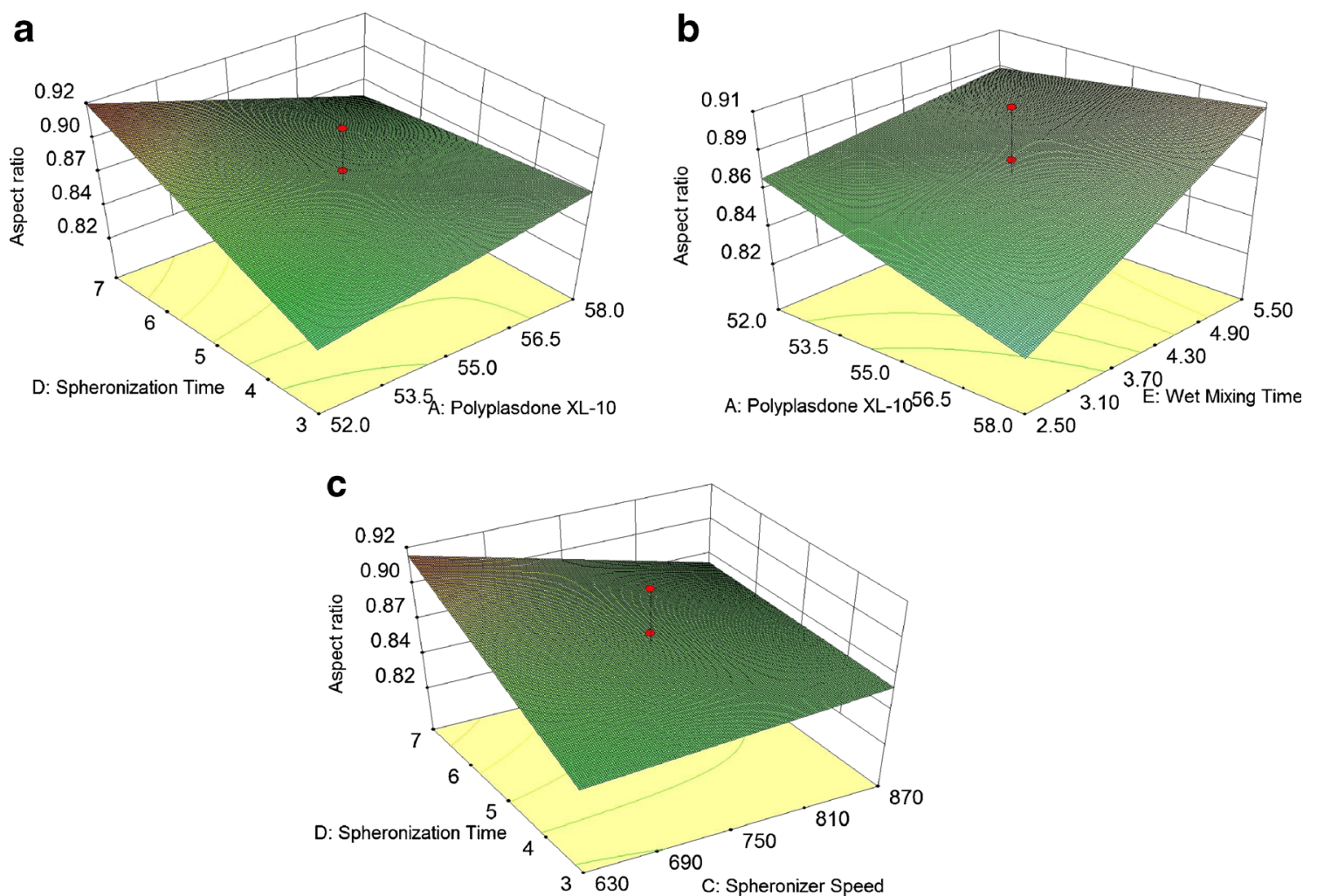

Fig. 7. Aspect ratio as a function of a Polyplasdone level and spheronization time, b Polyplasdone level and wet mixing time, and $\mathbf{c}$ Spheronizer speed and spheronizer time

With a higher level of crospovidone in the formulation, the water is distributed over a greater number of cross-linked polymer particles. If there is sufficient water to properly wet the crospovidone, it acts as an efficient binder [11] and, thereby, the friability of the pellets is decreased. Liquid bridges with sufficient dissolved solute(s) can dry to solid bridges that increase the integrity of the dried pellet [37]. This can be attributed to the increase in the caffeine content where caffeine acts as a solute that can form liquid bridges that turn to solid bridges when dried, thus increasing the strength of the pellets. In Fig. 8a, an increase in the spheronization time at low Polyplasdone level has little influence on the friability. On the other hand, with a high level of Polyplasdone in the formulation, a high spheronization time can diminish the friability. A high level of Polyplasdone suggests a more uniform distribution of water across the wetted mass during the wet massing step since

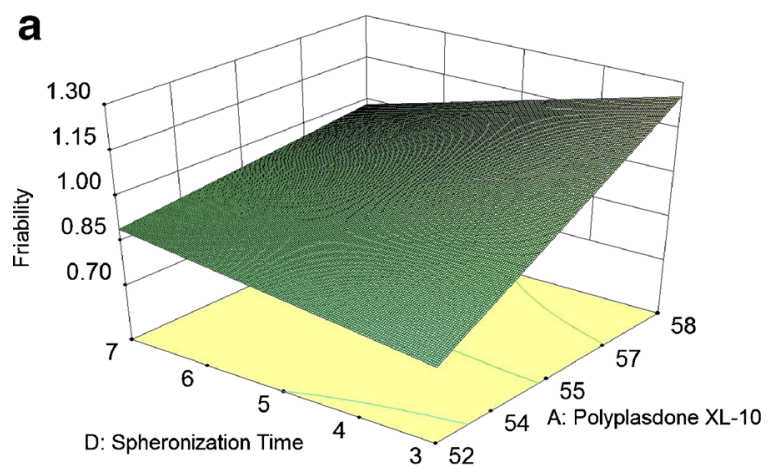

crospovidone takes up moisture readily. With an increase in the spheronization time, the wetted crospovidone in the extrudate particles can allow the formation of more liquid bridges as the material is experiencing compaction and the intermolecular distances are being reduced. Formation of solid bridges on drying of liquid bridges is critical to a rugged pellet product as demonstrated by its low friability.

Figure $8 \mathrm{~b}$ shows that, at a low wet mixing time, an increase in spheronizer speed results in an increase in the friability. The low wet mixing time does not allow even distribution of the water in the powder bed. In regions with lower hydration levels, liquid bridges are not formed and solid bridges do not form to enhance pellet integrity. In addition, the polymer is less plasticized such that more effective compaction of the extrudate with an increase in spheronizer speed does not occur to the same extent observed when the polymer

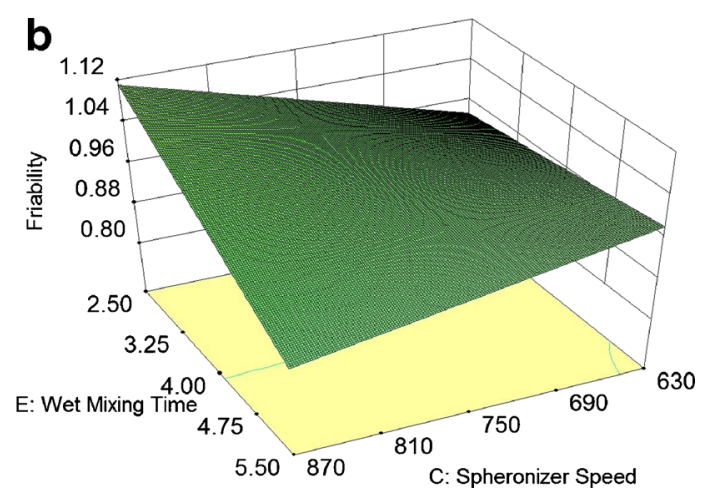

Fig. 8. Friability as a function of a Polyplasdone level and spheronization time and $\mathbf{b}$ spheronizer speed and wet mixing time 
Table IV. Predictability of the ANOVA Model Equations for Five of the Responses ${ }^{a}$

\begin{tabular}{lllllll}
\hline & Total yield $(\%)$ & Usable yield (\%) & Fines $(\%)$ & Average pellet diameter (mm) & Friability (\%) & Aspect ratio \\
\hline Predicted & 63.7 & 95.4 & 0.279 & 1.31 & 1.31 \\
Observed & $63.3+/-0.817^{b}$ & $96.3+/-0.874$ & $0.133+/-0.033$ & $1.35+/-0.125$ & $1.26+/-0.0614$ \\
Deviation & $0.628 \%$ low & $0.943 \%$ high & $50.7 \%$ low & $5.27 \%$ high & 0.920 \\
\hline
\end{tabular}

${ }^{a}$ Based on maximized total yield, usable yield, and aspect ratio with minimized fines and friability. Average pellet diameter was allowed to stay within the range observed across all the batches. This gave +1 for $\mathrm{A}, \mathrm{D}$, and $\mathrm{E}$ and -1 for $\mathrm{B}$ and $\mathrm{C}$ with a desirability of 0.974 . These factor levels match those for Run 14 for which the observed triplicate responses are noted

${ }^{b}+/-$ s.d. for $n=3$

is adequately plasticized. A reduction in compaction causes a lower bonding surface area within the individual pellet, and therefore, it lacks cohesivity. In contrast, at the high spheronizer speed, an increase in the wet mixing time reduces friability. This is a direct result of the adequate distribution of water across the powder bed that improves the plasticity and formation of liquid bridges in the now wetted mass.

\section{Dissolution}

As also observed by Verheyen et al. with pellets containing Kollidon ${ }^{\circledR}$ CL-M [19], the pellets disintegrated within a couple of minutes and fine white particles were seen floating throughout the medium. No further studies were conducted since it is evident that the pellets in the present study are an immediate release product.

\section{Predictability of the Model Equations}

Since curvature and lack of fit were not significant with any of the model equations for five responses, numerical optimization was accomplished with Design Expert ${ }^{\circledR}$ software. Optimized specifications were maximized total yield, usable yield and aspect ratio, and minimized fines and friability; average pellet diameter and sphericity were allowed to stay within the range observed across all of the batches. This gave +1 for $A, D$, and $E$, with -1 for B and C with a Desirability of 0.974. Using these levels in each of the response equations provides predicted values (Table IV). Three batches of pellets were prepared using the recommended factor levels, and the characteristics of pellets from those batches are presented as observed data in Table IV. With an exception of fines, no observed data deviation from its predicted value was greater than $6 \%$. It is evident that the model equation for five responses was able to predict the outcome under these optimized conditions.

\section{CONCLUSIONS}

By using experimental design, the positive or negative influence of formulation and process factors and their two-factor interactions on various pellet properties was revealed. Model equations derived using ANOVA were able to quantify the influence of these various factors. The hypothesis is thus supported. The ability of these equations to estimate well the responses was confirmed by producing three batches with the numerically optimized set of formulation and process factors and successfully predicting the responses using the model equations.

\section{ACKNOWLEDGMENTS}

The authors thank Richard Bruce from Johnson \& Johnson for his help with particle size and shape analyses.

\section{REFERENCES}

1. Kleinebudde P. The crystallite-gel-model for microcrystalline cellulose in wet-granulation, extrusion, and spheronization. Pharm Res. 1997;14(6):804-9.

2. Basit AW, Newton JM, Lacey LF. Formulation of ranitidine pellets by extrusion-spheronization with little or no microcrystalline cellulose. Pharm Dev Technol. 1999;4(4):499-505.

3. Brandl M, Magill A, Rudraraju V, Gordon MS. Approaches for improving the stability of ketorolac in powder blends. J Pharm Sci. 1995;84(10):1151-3.

4. George RC, Barbuch RJ, Huber EW, Regg BT. Investigation into the yellowing on aging of Sabrilâa $\hat{A}^{\circledR}$ tablet cores. Drug Dev Ind Pharm. 1994;20(19):3023-32.

5. Torres IA, Camacho AM. Solid state interactions of two new antineoplastic drugs (mitonafide and amonafide) and common tablet excipients in preformulation studies. Amsterdam: Elsevier; 1994.

6. Patel NK, Patel IJ, Cutie AJ, Wadke DA, Monkhouse DC, Reier GE. The effect of selected direct compression excipients on the stability of aspirin as a model hydrolyzable drug. Drug Dev Ind Pharm. 1988;14(1):77-98.

7. Signoretti EC, Dell'utri A, De Salvo A, Donini L. Compatibility study between clenbuterol and tablet excipients using differential scanning calorimetry. Drug Dev Ind Pharm. 1986;12(4):603-20.

8. Carstensen JT, Osadca M, Rubin SH. Degradation mechanisms for water-soluble drugs in solid dosage forms. J Pharm Sci. 1969;58(5):549-53.

9. Tripurasundari P, Prabhakar B. Review on the production of pellets via extrusion-spheronization exclusive of microcrystalline cellulose. Int J Pharm Rev Res. 2012;2(1):1-10.

10. Augsburger LL, Brzeczko AW, Shah U, Hahm HA. Super disintegrants: characterization and function. In: Swarbrick J, editor. Encyclopedia of pharmaceutical technology. 3rd ed. New York: Informa Healthcare USA, Inc.; 2007. p. 3553-67.

11. Liew CV, Gu L, Soh JL, Heng PW. Functionality of cross-linked polyvinylpyrrolidone as a spheronization aid: a promising alternative to microcrystalline cellulose. Pharm Res. 2005;22(8):1387-98.

12. Rowe RC, Sheskey PJ, Owen SC, Association AP, Library R. Handbook of pharmaceutical excipients. London: Pharmaceutical Press; 2003.

13. Saripella KK, Mallipeddi R, Neau SH. Crospovidone interactions with water. I. Calorimetric study of the effect of Polyplasdone particle size on its uptake and distribution of water. J Pharm Sci. 2014;103(2):669-75.

14. Saripella KK, Mallipeddi R, Neau SH. Crospovidone interactions with water. II. Dynamic vapor sorption analysis of the effect of Polyplasdone particle size on its uptake and distribution of water. Int J Pharm. 2014;475(1-2):174-80.

15. Sarkar S, Heng PW, Liew CV. Insights into the functionality of pelletization aid in pelletization by extrusion-spheronization. Pharm Dev Technol. 2013;18(1):61-72.

16. Sarkar S, Ang BH, Liew CV. Influence of starting material particle size on pellet surface roughness. AAPS PharmSciTech. 2014;15(1):131-9. 
17. Sarkar S, Wong TW, Liew CV. Importance of wet packability of component particles in pellet formation. AAPS PharmSciTech. 2013;14(3):1267-77.

18. Jain SP, Mehta DC, Shah SP, Singh PP, Amin PD. Melt-in-mouth pellets of fexofenadine hydrochloride using crospovidone as an extrusion-spheronisation aid. AAPS PharmSciTech. 2010.

19. Verheyen P, Steffens KJ, Kleinebudde P. Use of crospovidone as pelletization aid as alternative to microcrystalline cellulose: effects on pellet properties. Drug Dev Ind Pharm. 2009;35(11):1325-32.

20. Bühler V. Insoluble polyvinylpyrrolidone (crospovidone). Polyvinylpyrrolidone excipients for pharmaceuticals: povidone, crospovidone and copovidone. Berlin: Springer; 2005. p. 125-78.

21. Hileman GA, Goskonda SR, Spalitto AJ, Upadrashta SM. A factorial approach to high dose product development by an extrusion/spheronization process. Drug Dev Ind Pharm. 1993;19(4):483-91.

22. Hasznos L, Langer I, Gyarmathy M. Some factors influencing pellet characteristics made by an extrusion/spheronisation process. Part I.: effects on size characteristics and moisture content decrease of pellets. Drug Dev Ind Pharm. 1992;18(4):409-37.

23. Hileman GA, Upadrashta SM, Neau SH. Drug solubility effects on predicting optimum conditions for extrusion and spheronization of pellets. Pharm Dev Technol. 1997;2(1):43-52.

24. Marjolein DH. Development and optimization of a miniaturized extrusion-spheronization process for the formation of pure substance pellets. 2013.

25. Montgomery DC, Runger GC. Applied statistics and probability for engineers. 5th ed. Hoboken: Wiley; 2011. p. 562.

26. Osborne JW. Improving your data transformations: applying the box-cox transformation. Practic Assess Res Eval. 2010;15(12):1-9.
27. Hellén L, Yliruusi J, Merkku P, Kristoffersson E. Process variables of instant granulator and spheroniser: I. Physical properties of granules, extrudate and pellets. Int J Pharm. 1993;96(1-3):197-204.

28. Dressman JB. Comparison of canine and human gastrointestinal physiology. Pharm Res. 1986;3(3):123-31.

29. Glass GV, Hopkins KD. Statistical methods in education and psychology. 3rd ed. Boston: Allyn and Bacon; 1996. p. 674.

30. Crocker LM, Algina J. Introduction to classical and modern test theory. New York: Holt, Rinehart, and Winston; 1986. p. 527.

31. Kumar S, Das B, Raju SR. Formulation and evaluation of multiunit pellet system of venlafaxine hydrochloride. J Pharm Biomed Sci. 2012;18(5):1-12.

32. Wan LSC, Heng PWS, Liew CV. Spheronization conditions on spheroid shape and size. Int J Pharm. 1993;96(1-3):59-65.

33. Mallipeddi R, Saripella KK, Neau SH. Use of fine particle ethylcellulose as the diluent in the production of pellets by extrusion-spheronization. Saudi Pharm J. 2014;22(4):360-72.

34. Hasznos L, Langer L, Gyarmathy M. Some factors influencing pellet characteristics made by an extrusion/spheronization process. Part I. Effects on size characteristics and moisture content decrease of pellets. Drug Dev Ind Pharm. 1992;18:409-37.

35. Rowe RC. Spheronization: a novel pill-making process? Pharm Int,. 1985;S. 119-23.

36. Sinha VR, Agrawal MK, Kumria R, Bhinge JR. Influence of operational variables on properties of piroxicam pellets prepared by extrusion-spheronization: a technical note. AAPS PharmSciTech. 2007;8(1):20.

37. Endo Y, Kousaka Y, Onitsuka H. Adhesion force arising from solid salt bridge formed after drying of liquid bridge. KOKO. 1994;20(4):542-8. 